\title{
Fusobacterium nucleatum Interaction with Pseudomonas aeruginosa Induces Biofilm-Associated Antibiotic Tolerance via Fusobacterium adhesin A
}

Qian $\mathrm{Li}^{1}$, Lisi Tan ${ }^{2}$, Hongyan $\mathrm{Wang}^{2}$, Yurong Kou${ }^{1}$, Xiaoting Shi ${ }^{2}$, Shuwei Zhang ${ }^{2}$, and Yaping $\operatorname{Pan}^{1,2, *}$

1 Liaoning Provincial Key Laboratory of Oral Diseases, Department of Oral Biology, School and Hospital of Stomatology, China Medical University, Shenyang, Liaoning 110002, China

2 Liaoning Provincial Key Laboratory of Oral Diseases, Department of Periodontics, School and Hospital of Stomatology, China Medical University, Shenyang, Liaoning 110002, China

The supporting information includes two pages and one figure. 

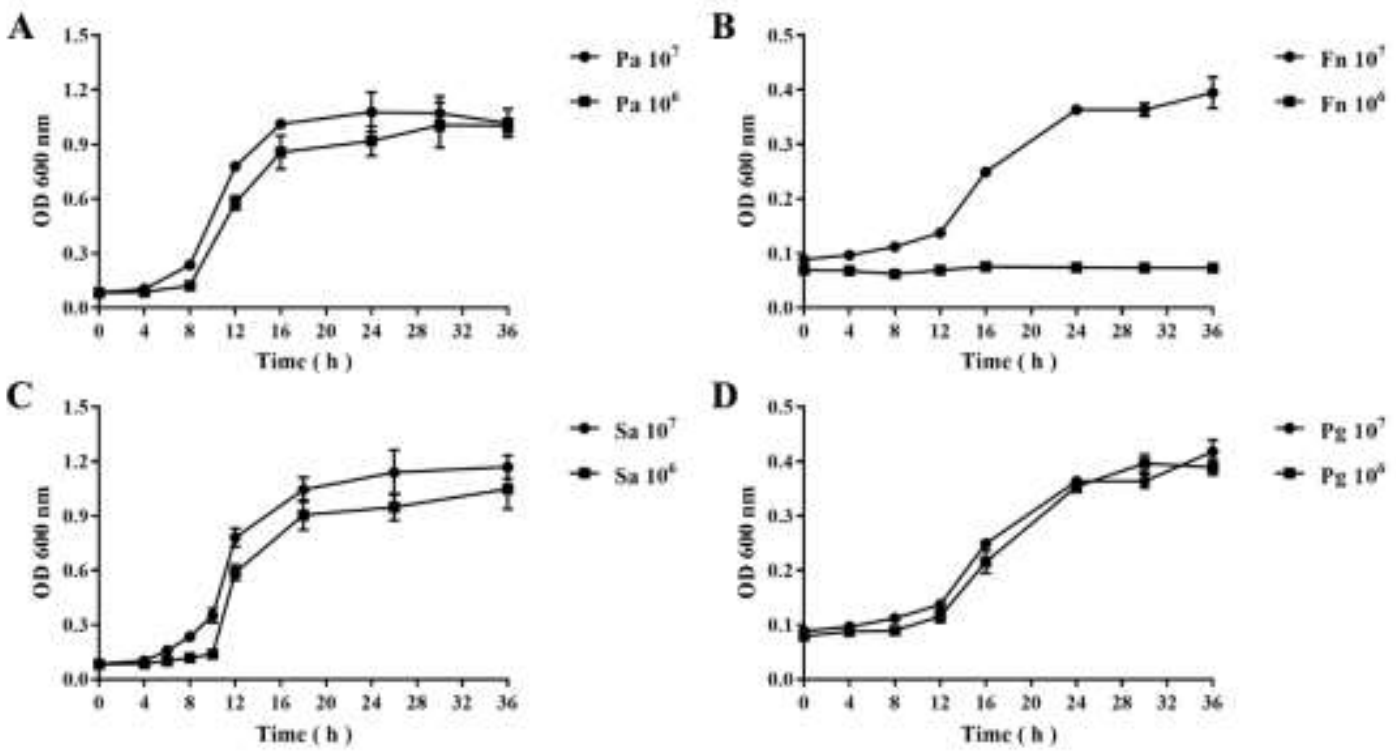

Figure S1. Bacterial Growth Curves. (A) Growth curves of P. aeruginosa in aerobic condition at an inoculation concentration of $1 \times 10^{6}$ or $1 \times 10^{7} \mathrm{CFU} / \mathrm{ml}$. (B) Growth curves of $F$. nucleatum in anaerobic condition at an inoculation concentration of $1 \times 10^{6}$ or $1 \times 10^{7} \mathrm{CFU} / \mathrm{ml}$. (C) Growth curves of $S$. aureus in aerobic condition at an inoculation concentration of $1 \times 10^{6}$ or $1 \times 10^{7} \mathrm{CFU} / \mathrm{ml}$. (D) Growth curves of $P$. gingivalis in anaerobic condition at an inoculation concentration of $1 \times 10^{6}$ or 1 $\times 10^{7} \mathrm{CFU} / \mathrm{ml}$. Each data was derived from three independent experiments with error bars as shown. Abbreviations: Pa, P. aeruginosa; Fn, F. nucleatum; Sa, S. aureus; Pg, P. gingivalis. 